\title{
Learning-Related Synaptic Growth Mediated by Internalization of Aplysia Cell Adhesion Molecule Is Controlled by Membrane Phosphatidylinositol 4,5-Bisphosphate Synthetic Pathway
}

\author{
Seung-Hee Lee, ${ }^{1}$ Jaehoon Shim, ${ }^{1}$ Sun-Lim Choi, ${ }^{1}$ Nuribalhae Lee, ${ }^{1}$ Chang-Hoon Lee, ${ }^{1}$ Craig H. Bailey, ${ }^{3}$ Eric R. Kandel, ${ }^{3,4}$ \\ Deok-Jin Jang, ${ }^{5}$ and Bong-Kiun Kaang ${ }^{1,2}$ \\ National Creative Research Initiative Center for Memory, Departments of ${ }^{1}$ Biological Sciences and ${ }^{2}$ Brain and Cognitive Sciences, College of Natural \\ Sciences, Seoul National University, Gwanak-gu, Seoul 151-747, Korea, ${ }^{3}$ New York State Psychiatric Institute, Kavli Institute for Brain Science, Department \\ of Neuroscience, Columbia University College of Physicians and Surgeons, New York, New York 10032, ${ }^{4}$ Howard Hughes Medical Institute, New York, New \\ York 10032, and ${ }^{5}$ Department of Applied Biology, College of Ecology and Environment, Kyungpook National University, Sangju-si, Kyeongbuk 742-711, \\ Korea
}

Long-term facilitation in Aplysia is accompanied by the growth of new synaptic connections between the sensory and motor neurons of the gill-withdrawal reflex. One of the initial steps leading to the growth of these synapses is the internalization, induced by 5-HT, of the transmembrane isoform of Aplysia cell-adhesion molecule (TM-apCAM) from the plasma membrane of sensory neurons (Bailey et al., 1992). However, the mechanisms that govern the internalization of TM-apCAM and how this internalization is coupled to the molecular events that initiate the structural changes are not fully understood. Here, we report that the synthesis of membrane phosphatidylinositol 4,5-bisphosphate $\left[\mathrm{PI}(4,5) \mathrm{P}_{2}\right]$, which is known to be mediated by a signaling cascade through Aplysia Sec7 protein (ApSec7) and phosphatidylinositol-4-phosphate 5-kinase type I $\alpha$ (PIP5KI $\alpha)$ is required for both the internalization of TM-apCAM and the initiation of synaptic growth during 5-HT-induced long-term facilitation. Pharmacological blockade of PI $(4,5) \mathrm{P}_{2}$ synthesis by the application of the inhibitor phenylarsine oxide blocked the internalization of apCAM. Furthermore, perturbation of the endogenous activation of ApSec7 and its downstream target PIP5KI $\alpha$ also blocked 5-HT-mediated internalization of TM-apCAM and synaptic growth. Finally, long-term facilitation was specifically impaired by blocking the ApSec7 signaling pathway at sensory-to-motor neuron synapses. These data indicate that the ApSec7/PIP5KI $\alpha$ signaling pathway is actively recruited during learning-related 5-HT signaling and acts as a key regulator of apCAM internalization associated with the formation of new synaptic connections during long-term facilitation.

\section{Introduction}

Synapses undergo structural changes in neurons that are engaged in memory-related long-term synaptic plasticity (Bailey and Kandel, 2008; Kandel, 2012). Synaptic growth and the subse-

Received April 17, 2012; revised Sept. 7, 2012; accepted Sept. 13, 2012.

Author contributions: S.-H.L., C.H.B., E.R.K., D.-J.J., and B.-K.K. designed research; S.-H.L., J.S., S.-L.C., N.L., and C.-H.L. performed research; S.-H.L., J.S., S.-L.C., N.L., and C.-H.L. contributed unpublished reagents/analytic tools; S.-H.L., J.S., S.-L.C., N.L., D.-J.J., and B.-K.K. analyzed data; S.-H.L., C.H.B., E.R.K., D.-J.J., and B.-K.K. wrote the paper. B.-K.K. was supported by the National Honor Scientist Program, the National Creative Research Initiative Program, and the World Class University program of the Korean Ministry of Science and Technology. D.-J.J., was supported by Kyungpook National University Research Fund, 2012. S.-H. L. and S.-L.C. were supported by a BK21 Research Fellowship from the Korea Ministry of Education and Human Resources Development. E.R.K. is supported by Howard Hughes Medical Institute and the Kavli Institute for Brain Science. E.R.K. and C.H.B. are also supported by the Simons Foundation.

The authors declare no competing financial interests.

Correspondence should be addressed to either of the following: Dr. Bong-Kiun Kaang, National Creative Research Initiative Center for Memory, Departments of Biological Sciences and Brain and Cognitive Sciences, College of Natural Sciences, Seoul National University, 599 Gwanangno, Gwanak-gu, Seoul 151-747, Korea; E-mail: kaang@snu.ac.kr; or Dr. Deok-Jin Jang, Department of Applied Biology, College of Ecology and Environment, Kyungpook National University, 386 Gajang-dong, Sangju-si, Kyeongbuk 742-711, Korea; E-mail: jangdj@knu.ac.kr.

DOI:10.1523/JNEUROSCI.1872-12.2012

Copyright $\odot 2012$ the authors $\quad 0270-6474 / 12 / 3216296-10 \$ 15.00 / 0$ quent formation of new functionally competent sensory neuron varicosities induced by the application of the modulatory neurotransmitter serotonin (5-HT) have been found to be critical for the persistence of facilitation beyond $24 \mathrm{~h}$ and thus are considered as key cellular mechanisms for the storage of long-term memory (Bailey et al., 2004; Lee et al., 2008). Synaptic growth during long-term facilitation (LTF) requires internalization of the transmembrane isoform of Aplysia cell adhesion molecule (TM-apCAM) (Mayford et al., 1992). Removal of TM-apCAM from the membrane surface is a necessary and permissive step for the initiation of learning-related synaptic growth (Bailey and Kandel, 2008). Immunoelectron microscopy has revealed that 5-HT stimuli that induce LTF also lead to the clathrin-mediated endocytosis of TM-apCAM from the surface membrane of sensory neurons (Bailey et al., 1992). The intracellular domain of TM-apCAM and its phosphorylation by MAPK is necessary for this process to occur (Bailey et al., 1997; Han et al., 2004), and CAMAP, a cytoplasmic binding partner of TM-apCAM, has been found recently to play an important role in stabilizing TMapCAM at the membrane surface (Lee et al., 2007). However, the 
A

A
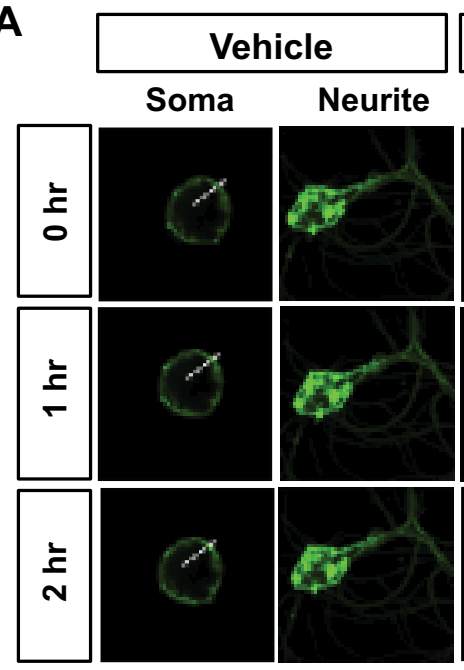

C

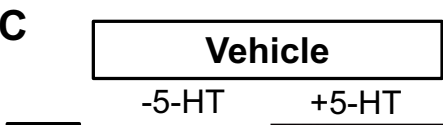

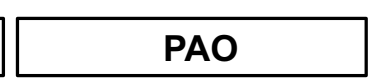
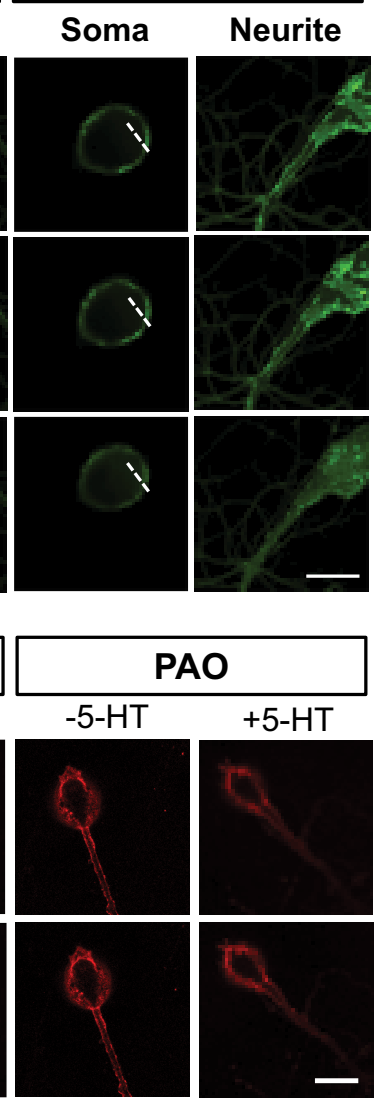

PAO

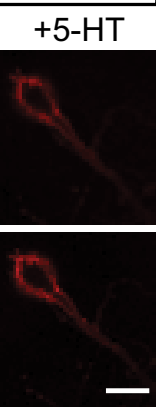

B
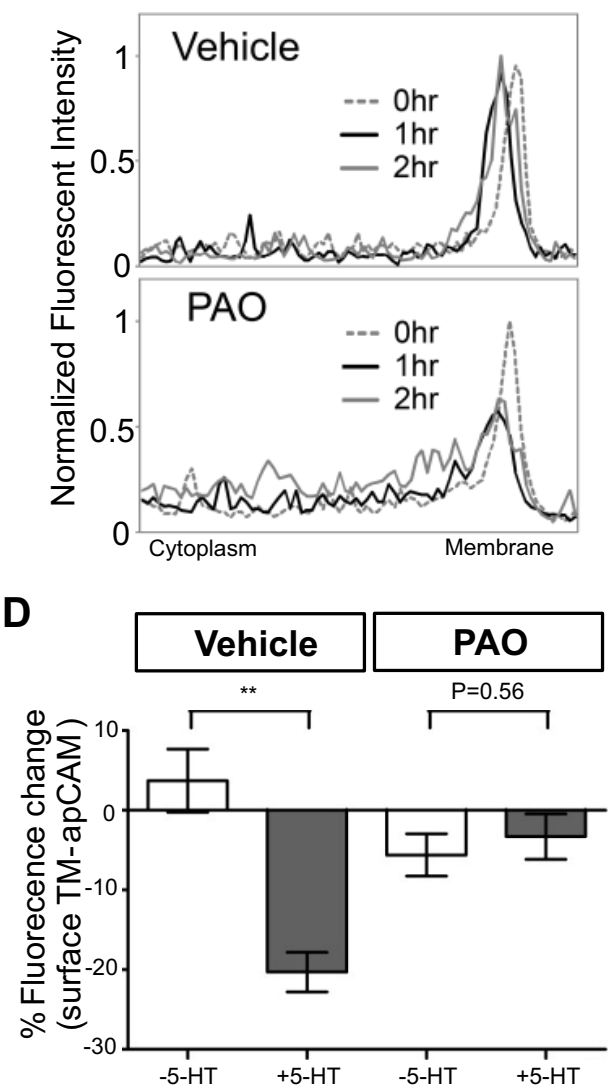

Figure 1. 5-HT-induced TM-apCAM internalization requires the PI(4,5)P2 synthetic pathway. $A$, Effect of PAO treatment on GFP_PHD localization in Aplysia sensory cells. Treatment with 20 $\mu$ M PAO but not the vehicle treatment induced cytosolic dispersion of membrane localized GFP-PHD. Scale bar, $40 \mu \mathrm{m}$. White dotted line, Area within which the level of fluorescent was measured. $\boldsymbol{B}$, Quantification of the dispersion of membrane GFP-PHD. Normalized fluorescent intensities in the area of soma corresponding to white dotted lines in $\boldsymbol{A}$ were plotted from the most cytoplasmic area (left of $x$-axis) to the plasma membrane (right of $x$-axis). Same area of the soma was measured at $0 \mathrm{~h}$ (gray dotted line), $1 \mathrm{~h}$ (black solid line), and $2 \mathrm{~h}$ (gray solid line). Note that the PA0 treatment decreased the level of membrane-localized GFP-PHD in $1 \mathrm{~h}$. C, Live-cell immunostaining of surface TM-apCAM. Either vehicle (DMSO) or PA0 (20 $\mu \mathrm{m})$ was concurrently treated with 5-HT. Images were taken at $\mathrm{h}$ (top) and $1 \mathrm{~h}$ after 5 -HT treatment (bottom) in the same cell. Scale bar, $40 \mu \mathrm{m}$. D, Percentage fluorescence changes of surface-stained TM-apCAM without ( -5 -HT) or with 5-HT treatment $(+5-\mathrm{HT})$ in the presence of vehicle or PAO (vehicle, $-5-\mathrm{HT}, n=6$; vehicle, $+5-\mathrm{HT}, n=10 ; \mathrm{PAO},-5-\mathrm{HT}, n=9 ; \mathrm{PAO},+5-\mathrm{HT}, n=9$; mean $\pm \mathrm{SEM}$; ${ }^{* *} p<0.01, \mathrm{unpaired} t$ test).

molecular pathway whereby 5-HT signaling initiates the internalization of TM-apCAM at the plasma membrane of the sensory neurons to induce the synaptic growth that accompanies LTF is still not known.

It is generally thought that lipid signaling is critical for the membrane dynamics and subsequent protein internalization from the surface membrane (Simons and Gerl, 2010). For example, membrane phosphatidylinositol 4,5-bisphosphate $\left[\mathrm{PI}(4,5) \mathrm{P}_{2}\right]$, which is mostly generated from phosphatidylinositol 4-phosphate by phosphatidylinositol-4-phosphate 5-kinases (PIP5Ks) (Yin and Janmey, 2003; Rameh et al., 1997; Jang et al., 2009), regulates the rearrangement of actin cytoskeletons and endo-exocytosis by binding to transmembrane or adaptor proteins and recruiting downstream signaling pathways (Raucher et al., 2000; Di Paolo and De Camilli, 2006). The major upstream molecules of PIP5Ks are the ADP ribosylation factor (ARF) family of small GTPases and their guanine nucleotide exchange factors (GEFs), the Sec7 protein family (Honda et al., 1999; Hernández-Deviez et al., 2004; Funakoshi et al., 2011; Casanova, 2007). In Aplysia neurons, overexpression of msec7-1, a mammalian homolog of Sec7 proteins, produces extensive neurite outgrowth, increases the number of synaptic varicosities, and enhances synaptic transmission (Huh et al., 2003). During LTF, application of 5-HT induces rapid and permanent changes in the structure of the plasma membrane and internal membrane component of the cytoplasm, leading to synaptic growth and synaptic facilitation (Bailey et al., 2004). We therefore tested the possible role of the $\mathrm{PI}(4,5) \mathrm{P}_{2}$ synthetic pathway in regulating the internalization of TM-apCAM and initiating synaptic growth. We found that $\mathrm{PI}(4,5) \mathrm{P}_{2}$ synthesis and its major upstream signaling pathway of the Aplysia Sec7 protein (ApSec7)/PIP5KI $\alpha$ pathway are critical for the internalization of TM-apCAM, synaptic growth, and facilitation. Our data support the idea that 5-HT-induced LTF requires activation of the ApSec7/PIP5KI $\alpha$ signaling pathway to induce membrane $\mathrm{PI}(4,5) \mathrm{P}_{2}$ synthesis, which is important for the internalization of TM-apCAM, synaptic growth, and the induction of LTF.

\section{Materials and Methods}

Cloning of ApSec7 and generation of ApSec7E159K mutant. To identify the Sec7 proteins in Aplysia, the standard hybridization screening of Aplysia kurodai CNS cDNA library was performed using the ${ }^{32} \mathrm{P}$-labeled Sec7 domain of msec7-1 as a probe (GenBank accession number JX458713). Based on the nucleotide sequences of identified clones, recombinant PCR was used to generate a dominant-negative (DN) mutant of ApSec7, which has null catalytic activity of GEF at the conserved sec7 domain. The 


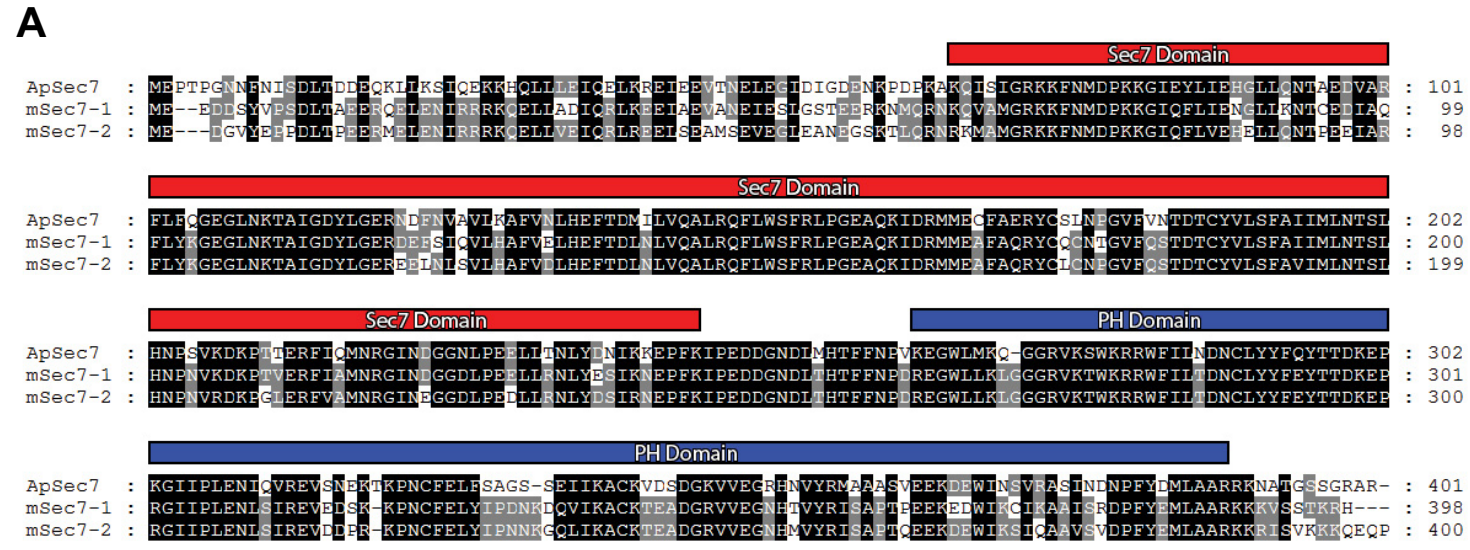

B

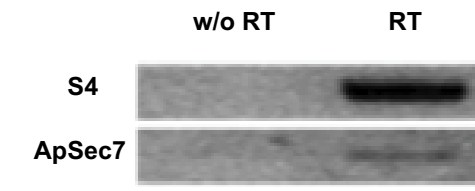

D
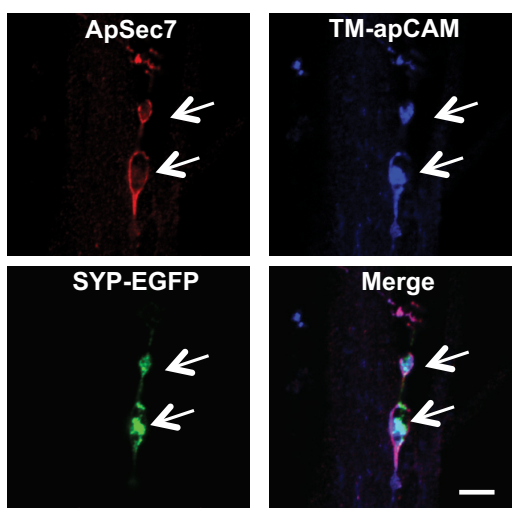

C
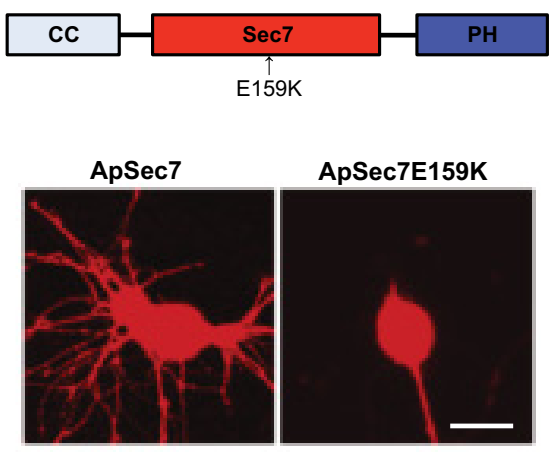

ApSec7E159K

$\mathbf{E}$

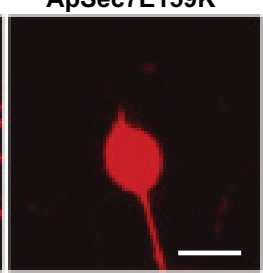

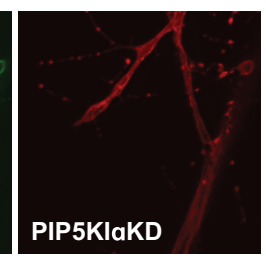

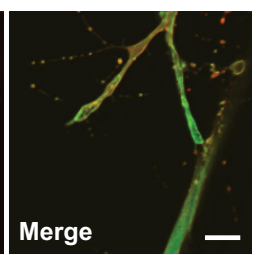

Figure 2. Cloning and characterization of ApSec7 in Aplysia sensory neurons. A, Multiple sequence alignment of ApSec7 with mammalian mSec7-1 and mSec7-2. Red bar, Homologous Sec7 domain; blue bar, PH domain. B, RT-PCR analysis in cultured sensory neurons. Both a housekeeping gene $S 4$ and ApSec7 mRNAs are present in cultured sensory neurons. $C$, Expression of ApSec7 or ApSec7E159K in Aplysia sensory neurons. Top, Schematic for ApSec7 and the targeted mutation of E159K. Bottom, Immunostaining for the overexpressed ApSec7 or ApSec7E159K (red). Note that wild-type ApSec7, but not the mutant ApSec7E159K, enhanced neuritogenesis. Scale bar, $40 \mu \mathrm{m}$. D, E, Subcellular localization of ApSec7/PIP5KI $\alpha / T M$-apCAM in Aplysia sensory neurons. D, Coexpression of ApSec7 (red), TM-apCAM (blue), and SYP-EGFP (green) in the distal neurites and varicosities of Aplysia sensory neurons contacting motor neurons. Merge shows colocalization (pink) between ApSec7 and TM-apCAM at the membrane of the varicosities (arrows) as well as colocalization (cyan) between TM-apCAM and SYP-EGFP. E, Colocalization (yellow) of ApSec7 (green) and PIP5KI $\alpha$ KD (red) in the distal neurites of Aplysia sensory neurons. Scale bars, $20 \mu \mathrm{m}$.

mutant fragments of ApSec7-E159K was generated by recombinant PCR using specific sense or antisense primers (sense primer containing E159K, 5' -CCCGGTAAAGCACAGAAGA-3'; antisense primer containing E159K, 5'-TCTTCTGTGCTTTACCGGG-3'). The PCR products containing mutation (E159K) in ApSec7 amino acid residues were subcloned into HindIII-XbaI digested pNEX $\delta$ vector.

$R T-P C R$. Total RNAs were extracted from Aplysia central ganglia using Trizol Reagent. The cDNA was synthesized by Superscript III reverse transcriptase with oligo-dT or random hexamer as a primer. The cDNA was used as templates for PCR reactions for ApSec7 (sense, 5' -ATGAAG CAAGGTGTCCCTAAAAT- $3^{\prime}$; antisense, $5^{\prime}$-CTTTCCGTCCGAGTCA ACTTT-3') and S4 (sense, 5'-GACCCTCTGGTG-AAGGTGAA-3'; antisense, $5^{\prime}$-TGGACAGCTTCACACCTTTG-3'). Amplification was performed for $28-35$ cycles $\left(94^{\circ} \mathrm{C}, 15 \mathrm{~s} ; 52^{\circ} \mathrm{C}, 15 \mathrm{~s} ; 72^{\circ} \mathrm{C}, 30 \mathrm{~s}\right)$. PCR products were visualized on $1 \%$ agarose gel.

Double-strand RNA synthesis. Partial sequences of ApSec7 containing Sec7 and pleckstrin homology domains (PHDs) cut by HindIII and XbaI were subcloned into a pLITMUS28i vector (New England Biolabs). Double-strand RNA (dsRNA) of ApSec7 and firefly luciferase were syn- thesized by an in vitro transcription using MEGAscript RNAi kit (Ambion) according to the protocol of the manufacturer. The resulting dsRNA was dissolved in a microinjection buffer containing $0.1 \%$ fast green, $10 \mathrm{~mm}$ Tris-Cl, pH 7.3, and $100 \mathrm{~mm} \mathrm{KCl}$.

Microinjection into Aplysia sensory neurons cocultured with motor neurons. Neurons were isolated from ganglia of Aplysia kurodai and cultured in the poly-L-lysine-coated plates. Microinjection into Aplysia neurons was as described previously (Kaang, 1996). Sensory neurons in sensorimotor neuron cocultures were microinjected with an injection solution containing $0.5 \mu \mathrm{g} / \mu \mathrm{l}$ pNEX $\delta$-EGFP and $0.5 \mu \mathrm{g} / \mu \mathrm{l}$ dsRNA of ApSec7, pNEX $\delta$-ApSec7, pNEX $\delta$-ApSec7E159K, or pNEX $\delta$-PIP5KI $\alpha$ KD (GenBank accession number U78575; Loijens and Anderson, 1996; Coppolino et al., 2002) $24 \mathrm{~h}$ before the 5-HT treatment. Successful RNA or DNA microinjections were determined $24 \mathrm{~h}$ after the microinjection into the sensory neurons by the expression of GFP fluorescence.

Electrophysiological recording. All the recordings were performed as described previously (Lee et al., 2003; Jang et al., 2010, 2011). Lateral funiculus motor neurons, which are cocultured with sensory neurons, were impaled with glass microelectrodes to measure the EPSP. To ana- 
A

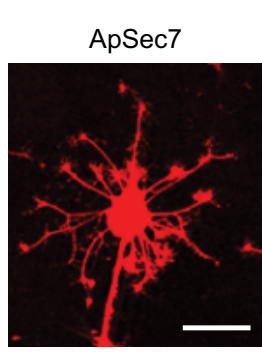

ApSec7 + PIP5KlaKD
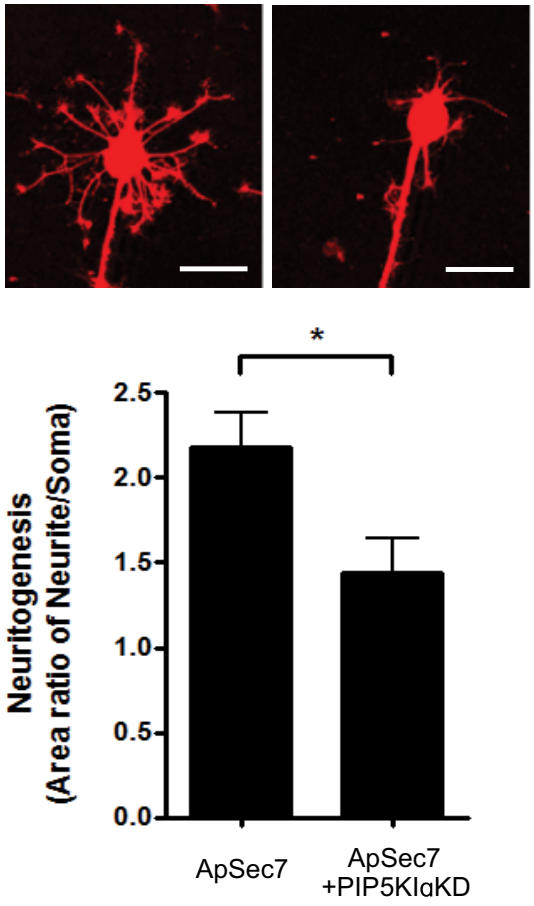

B

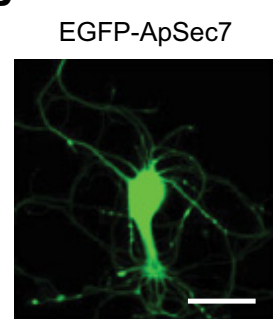

EGFP-ApSec7 + DN-Cdc42

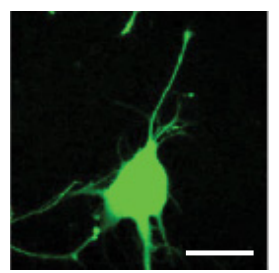

EGFP-ApSec7 + DN-Rac1

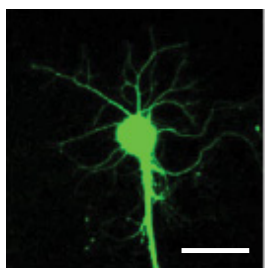

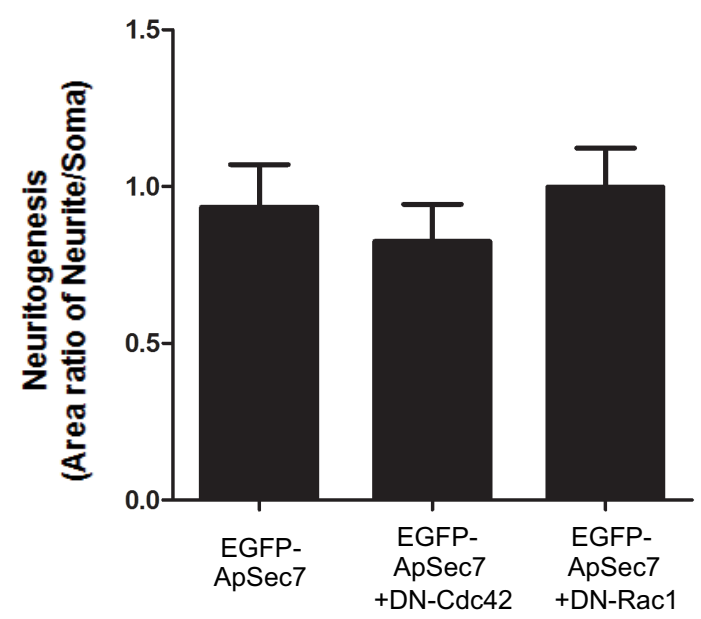

Figure 3. Neuritogenesis induced by ApSec7 was significantly blocked by PIP5KI $\alpha$ KD but not by DN-Cdc42 or DN-Rac1. A, Top, Representative images of ApSec7-mediated neuritogenesis in sensory neurons expressed with ApSec7 (left) or ApSec7 with PIP5KI $\alpha$ KD (right). Scale bars, $50 \mu \mathrm{m}$. Bottom, Ratio between area of neurite and soma (ApSec7, $n=38 ;$ ApSec7 + PIP5KI $\alpha$ KD, $n=$ 39 ; mean \pm SEM; ${ }^{*} p<0.05$, unpaired $t$ test). Coexpression of PIP5KI $\alpha$ KD significantly suppressed the neuritogenesis. $B$, Top, Representative images of Aplysia sensory neurons overexpressed with EGFP-ApSec7 (left), EGFP-ApSec7 with DN-Cdc42 (middle), or EGFP-ApSec7 with DN-Rac1 (right). Scale bars, $50 \mu \mathrm{m}$. Bottom, Ratio between area of neurite and soma (EGFP-ApSec7, $n=19$; EGFP-ApSec7+DN-Cdc42, $n=13 ;$ EGFP-ApSec7+DN-Rac1, $n=16$; mean \pm SEM; $p=0.67$, one-way ANOVA).

lyze the effect of knockdown or overexpression of ApSec7 on LTF, the initial EPSP was measured $24 \mathrm{~h}$ after the microinjection, and then five pulses of 5-HT $(10 \mu \mathrm{M})$ were applied with an interstimulus interval (ISI) of $15 \mathrm{~min}$. Twenty-four hours after the 5-HT treatment, final EPSP was measured. For depression recording, series of EPSP in response to 10 repeated stimuli with ISIs of $20 \mathrm{~s}$ were measured.

Immunocytochemistry and analysis of the neuritogenesis. cDNAs of ApSec7, PIP5KI $\alpha$ KD, DN-ApCdc42, or DN-ApRacl were subcloned into the Aplysia neuronal expression vector $\mathrm{pNEX} \delta$ with flag or $\mathrm{HA}$ tag on their $\mathrm{C}$ terminus. These constructs were expressed in the Aplysia sensory neurons by microinjection. Neurons were fixed and immunostained using anti-flag M2 antibodies (Abs) (Sigma) or anti-ApSec7 antiserum $36 \mathrm{~h}$ after expression. Fluorescent and differential interference contrast (DIC) images were taken under laser scanning confocal microscope (LSM510; Carl Zeiss). Total area of neurons including newly synthesized neurites were measured by total number of pixels of stained neurons based on the fluorescence images. The threshold of fluorescent signal was determined based on the actual neurite morphology in the DIC images to match the fluorescent signal within the neurite areas. Soma size was measured based on the DIC images. Net area of neurites was calculated as follows: pixel number of total area - pixel number of soma area.

Varicosity measurement. To measure total number of varicosities together with synaptophysin (SYP)-EGFP-filled varicosities, pNEX3-SYP-EGFP was coinjected with $\mathrm{pNEX} \delta-\mathrm{ApSec} 7$ or pNEX $\delta$ control vector into sensory neurons cocultured with motor neurons. Thirty-six hours after expression, the total morphology of sensory neurons was imaged by microinjecting the whole-cell fluorescence dye Alexa Fluor 594. Green and red fluorescence images were taken under the fluorescence microscope (Olympus IX51). To measure changes in total number of varicosities during LTF, we injected pNEX3-EGFP together with pNEX $\delta$-ApSec7E159K or with pNEX $\delta$ PIP5KI $\alpha$ KD. Twenty-four hours after injection, each sensory neuron was imaged $3 \mathrm{~h}$ before and $24 \mathrm{~h}$ after five pulses of 5-HT treatment under the confocal microscope. $Z$-stack images were taken to measure total number of varicosities. Identification of varicosities was as reported previously (Glanzman et al., 1990; Kim et al., 2003).

apCAM internalization assay. Imaging and quantification of apCAM internalization in live sensory neurons were performed as described previously (Han et al., 2004; Lee et al., 2007). Briefly, cultured sensory neurons were overexpressed with TM-apCAM that was tagged with HA at the extracellular domain of apCAM. Twenty-four hours after the expression, cultures were rinsed with perfusion media [1:1 mixture of isotonic L15 and artificial seawater (ASW)] and incubated with the anti-HA Ab 12CA5 (first Ab; 1:100; HA-7; Sigma) for 40 min. After being rinsed with perfusion media, cultures were incubated with the Cy3-conjugated antimouse IgG (second Ab; 1:100) for $40 \mathrm{~min}$. Then cultures were rinsed with perfusion media, and fluorescently labeled apCAM on the surface of sensory neurons was imaged under the confocal microscope (LSM510; Carl Zeiss). Immediately after the cells were viewed, each culture was treated with either control solution (isotonic L15 plus ASW) or $10 \mu \mathrm{M}$ 5 -HT in control solution for $1 \mathrm{~h}$. Changes in fluorescence intensity in each cell before and after 5-HT treatment were analyzed.

\section{Results}

Membrane $\mathrm{PI}(4,5) \mathrm{P}_{2}$ synthesis is required for the internalization of TM-apCAM

To begin to elucidate the molecular mechanisms that underlie apCAM internalization, we tested a possible role of membrane $\mathrm{PI}(4,5) \mathrm{P}_{2}$ synthesis (Czech, 2003) after the application of 5-HT. Phenylarsine oxide (PAO) has been found to inhibit $\mathrm{PI}(4,5) \mathrm{P}_{2}$ synthesis at the plasma membrane by blocking the activity of PIP5K, which catalyzes synthesis of $\mathrm{PI}(4,5) \mathrm{P}_{2}$ on the lipid plasma membrane (Wiedemann et al., 1996; Doughman et al., 2003). In Aplysia sensory neurons, $20 \mu \mathrm{M}$ PAO treatment induced cytosolic 

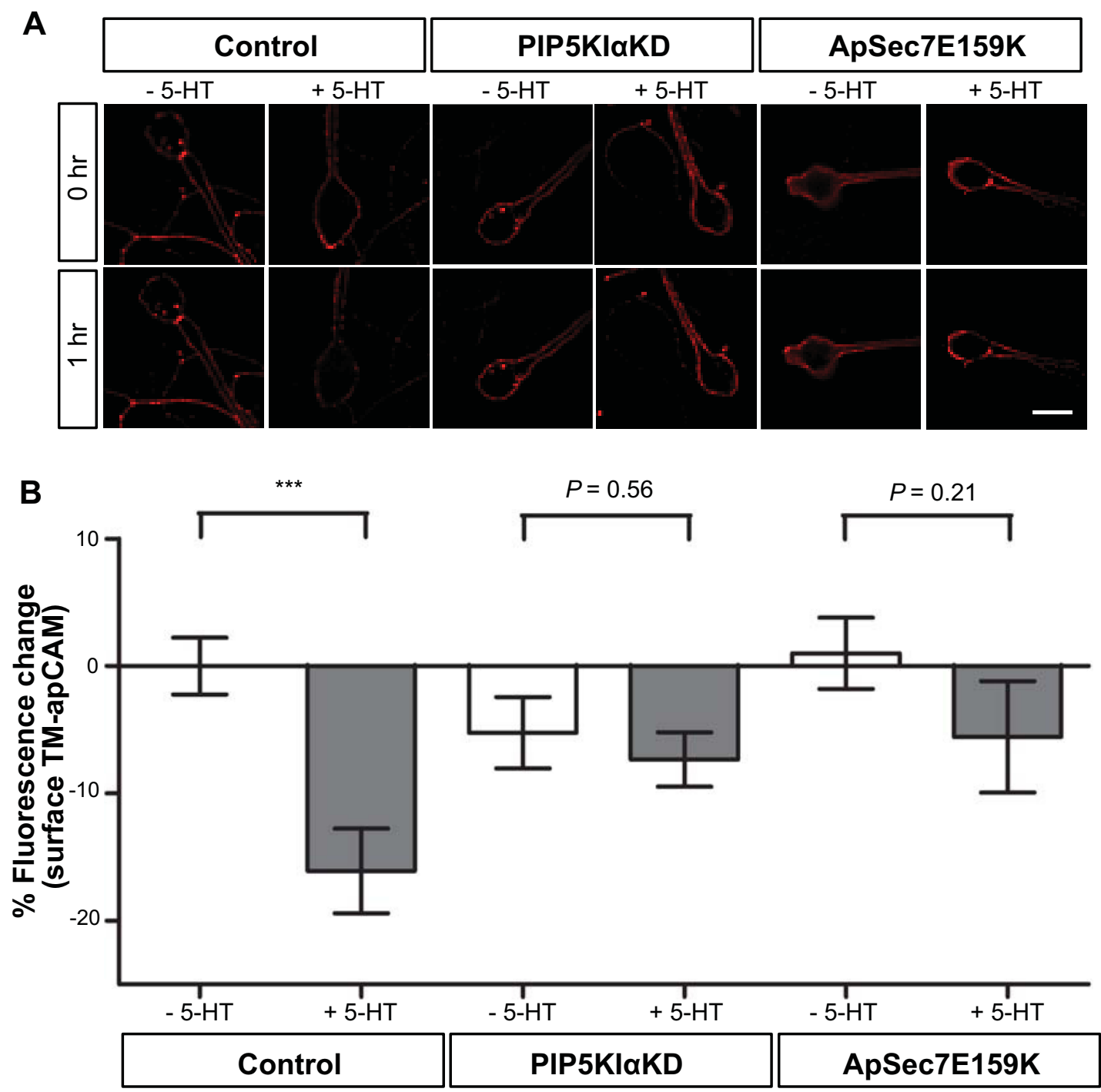

Figure 4. ApSec7/PIP5KI $\alpha$ signaling pathway is required for the 5-HT-induced internalization of TM-apCAM in Aplysia sensory neurons. A, Live-cell immunostaining of surface TM-apCAM (red). Cells were overexpressed with blank vector (Control), PIP5KI $\alpha$ KD, or ApSec7E159K. B, Percentage florescence changes of surface-strained TM-apCAM without ( -5 -HT) or with 5-HT treatment $(+5-\mathrm{HT}$ ) in the overexpression condition as $\boldsymbol{A}$ (control, -5 - HT, $n=18$; control, $+5-\mathrm{HT}, n=19$; PIP5KI $\alpha \mathrm{KD},-5-\mathrm{HT}, n=17$; PIP5KI $\alpha \mathrm{KD},+5$-HT, $n=21$; ApSec7E159K, -5 -HT, $n=13$; ApSec7E159K, $+5-\mathrm{HT}, n=21$; mean \pm SEM; ${ }^{* * *} p<0.001$, unpaired $t$ test).

dispersion of the membrane $\mathrm{PI}(4,5) \mathrm{P}_{2}$ indicator GFP-PHD [phospholipase C $\delta 1$ (PLC $\delta 1$ )] (Fig. 1 A,B) (Di Paolo and De Camilli, 2006). These data indicate that PAO blocks membrane $\mathrm{PI}(4,5) \mathrm{P}_{2}$ synthesis and depletes $\mathrm{PI}(4,5) \mathrm{P}_{2}$ from the plasma membrane in cultured Aplysia neurons. In the presence of PAO, 5-HTinduced internalization of TM-apCAM was totally abolished in Aplysia sensory neurons (Fig. 1C,D). Thus, the supplement of $\mathrm{PI}(4,5) \mathrm{P}_{2}$ by PIP5K at the plasma membrane is required for 5-HT-induced TM-apCAM internalization.

ApSec7 and PIP5KI $\alpha$ is a potential upstream signaling molecule of membrane $\mathrm{PI}(4,5) \mathrm{P}_{2}$ synthesis in Aplysia sensory neurons

Because we have found that PIP5K activity is critical for the 5-HT-mediated internalization of TM-apCAM, we hypothesized that the major upstream activator of PIP5Ks, ARF small GTPase and its GEF Sec7 proteins (Honda et al., 1999; Hernández-Deviez et al., 2004; Funakoshi et al., 2011; Casanova, 2007), might also be important for the internalization of TM-apCAM. We, therefore, cloned ApSec7 by conventional cDNA library screening as a potential upstream target molecule that modulates the ARF/PIP5K signaling pathway and synthesis of $\mathrm{PI}(4,5) \mathrm{P}_{2}$ (see Materials and Methods; Fig. 2). ApSec7 is homologous to mammalian Sec7 proteins (msec7-1) and contains a highly conserved domain structure with $\mathrm{N}$-terminal coiled-coil domain, middle Sec7 domain, and C-terminal PHD (Fig. 2A) (Telemenakis et al., 1997; Cherfils et al., 1998; Mossessova et al., 1998). We confirmed the presence of ApSec7 mRNA transcript in the total RNA extract from isolated cultured sensory neurons (Fig. $2 B$ ). The Sec7 domain is the catalytic domain for the GDP-GTP exchange, and the point mutation substituting highly conserved glutamate (E) in the Sec7 domain to lysine (K) leads to an impairment of GEF activity in Sec7 proteins but preserves their ARF-binding property (Cherfils et al., 1998; Mossessova et al., 1998). In Aplysia neurons, overexpression of the mouse homolog of sec7 protein homolog, msec7-1, induced extensive neuritogenesis, and this effect was not observed when the null mutant msec7-1, msec7-1 E157K, was overexpressed (Huh et al., 2003). Similarly, enhanced 
A

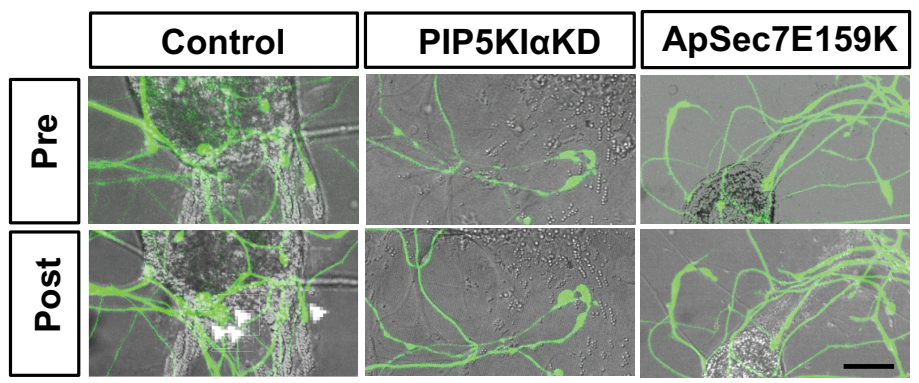

B

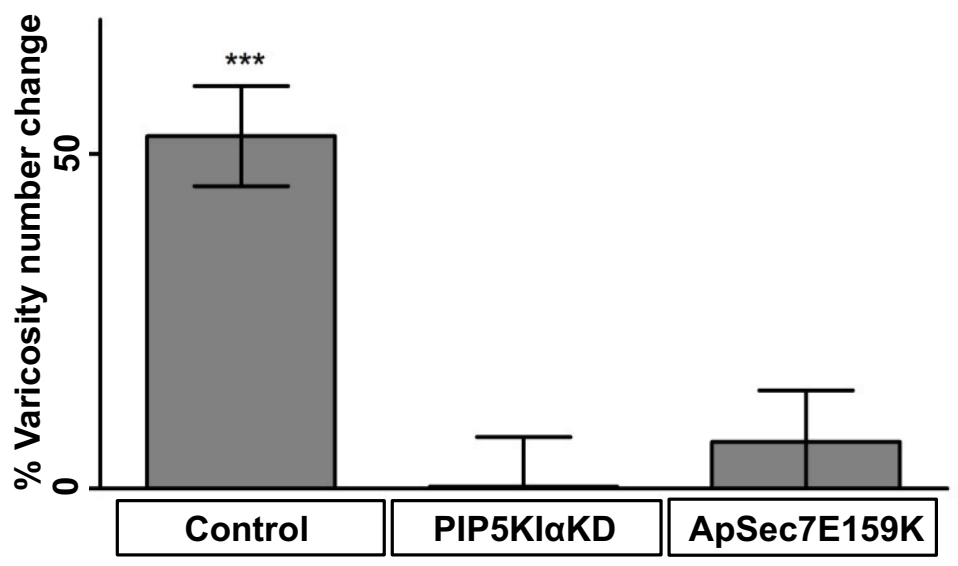

Figure 5. ApSec7/PIP5KI $\alpha$ signaling pathway is required for the 5 -HT-induced synaptic growth in Aplysia sensory-to-motor neuron synapses. $A$, Varicosity number changes after 5 -HT treatment. Varicosities of sensory neurons contacting major axons of the postsynaptic motor neurons were counted in the sensory neurons expressing only EGFP (Control), EGFP with PIP5KI $\alpha$ KD, or EGFP with ApSec7E159K. Green, EGFP-filled neurites and varicosities of sensory neurons. Gray, DIC images of the areas in which the varicosities were measured. Arrowheads, Newly synthesized varicosities. Pre, $3 \mathrm{~h}$ before 5 - $\mathrm{HT}$ treatment; Post, $24 \mathrm{~h}$ after five pulses of 5 -HT treatment. Scale bar, $40 \mu \mathrm{m}$. B, Percentage change of the varicosity number (control, $n=18$; PIP5KI $\alpha \mathrm{KD}, n=13$; ApSec7E159K, $n=11$; mean \pm SEM; ${ }^{* * *} p<0.001$, one-way ANOVA followed by Dunnett's multiple comparison test).

critical for initiating TM-apCAM internalization. Furthermore, overexpression of ApSec7E159K, like PIP5KI $\alpha$ KD, significantly blocked TM-apCAM internalization after exposure to 5-HT (Fig. $4 A, B$; ApSec7E159K). These results indicate that ApSec7 as well as PIP5KI $\alpha$ are critical mediators for TM-apCAM internalization. Collectively, our data support the idea that 5-HT-induced TM-apCAM internalization is mediated by $\mathrm{PI}(4,5) \mathrm{P}_{2}$ synthesis via the ApSec7/ PIP5KI $\alpha$ signaling pathway in Aplysia sensory neurons.

\section{ApSec7/PIP5KI $\alpha$ signaling pathway is required for synaptic growth during LTF}

Internalization of TM-apCAM is critical for the learning-related synaptic growth in cultured sensory neurons forming synapses with motor neurons in vitro (Bailey et al., 1992; Mayford et al., 1992; Han et al., 2004). We next tested whether ApSec7/PIP5KI $\alpha$ signaling activation was also required for 5-HT-induced synaptic growth. We expressed either PIP5KI $\alpha$ KD or ApSec7E159K in the presynaptic sensory neuron cocultured with a postsynaptic motor neuron and counted the total number of sensory neuron varicosities formed on the initial segment and major axons of the motor neuron before and $24 \mathrm{~h}$ after five pulses of

ApSec7 signaling by overexpression of ApSec7 induced massive neuritogenesis from somata of cultured sensory neurons, whereas overexpression of the null mutant ApSec7E159K did not induce any neuritogenesis (Fig. 2C). ApSec7 was colocalized at the plasma membrane with TM-apCAM (Fig. 2D), and this coincided with where $\mathrm{PI}(4,5) \mathrm{P}_{2}$ was located (Fig. $1 A$ ).

As an independent approach to blocking the activity of the PIP5K, we targeted expression of a DN [kinase-dead (KD)] form of human PIP5KI $\alpha$ (PIP5KI $\alpha$ KD) (Coppolino et al., 2002) into cultured sensory neurons. Ectopically expressed human PIP5KI $\alpha$ KD was highly colocalized with the ApSec7 at the membrane (Fig. 2E). Furthermore, the enhanced neuritic outgrowth by overexpressed ApSec7 was significantly suppressed by coexpression of PIP5KI $\alpha \mathrm{KD}$ (Fig. 3A), suggesting that PIP5KI $\alpha$ is one of the major downstream signaling molecules of ApSec7. Neither DN-ApCdc42 nor DN-ApRac1 suppressed the neuritogenesis in ApSec7-expressed neurons (Fig. 3B), supporting the specific and critical role of PIP5KI $\alpha$ as a downstream signaling molecule of ApSec7 in Aplysia sensory neurons.

\section{ApSec7/PIP5KI $\alpha$ signaling pathway is required for the internalization of TM-apCAM}

We next tested the role of ApSec7/PIP5KI $\alpha$ in regulating internalization of TM-apCAM. Similar to results from PAO treatment (Fig. $1 C, D$ ), overexpression of PIP5KI $\alpha \mathrm{KD}$ significantly blocked 5-HT-induced TM-apCAM internalization when compared with control sensory neurons without expression (Fig. 4A, B; control vs PIP5KI $\alpha \mathrm{KD}$ ). These data further support the idea that the signaling pathway for synthesis of membrane $\mathrm{PI}(4,5) \mathrm{P}_{2}$ is
5-HT treatment that induces LTF (Kandel, 2001; Kim et al., 2003; Bailey and Kandel, 2008). In the control cells, repeated pulses of 5 -HT induced a significant increase in the total number of varicosities. This synaptic growth was completely blocked by expression of either PIP5KI $\alpha$ KD or ApSec7E159K (Fig. 5A, B). Thus, in addition to its role in internalization of TM-apCAM, ApSec7/ PIP5KI $\alpha$ is also a key mediator for the 5-HT-induced formation of new sensory neuron varicosities in Aplysia.

ApSec7 signaling pathway is critical for the induction of LTF To further test whether inhibition of synaptic growth by disrupting the ApSec7/PIP5KI $\alpha$ signaling pathway directly affects LTF, we manipulated ApSec7 signaling in the presynaptic sensory neurons. First, we attempted to disrupt the ApSec7 signaling pathway by overexpressing ApSec7E159K in sensory neurons. We found that short-term facilitation (STF) was induced normally after acute exposure to 5-HT, whereas LTF was blocked at the same synapses at $24 \mathrm{~h}$ after repeated pulses of 5-HT (Fig. 6). In Aplysia neurons, targeted knockdown of specific genes can be achieved by microinjection of sequence-specific dsRNAs (Lee et al., 2001). Based on the newly cloned gene ApSec7, we generated dsRNA against partial sequences ( $~ 500 \mathrm{nt})$ of Sec7-PHD of ApSec7 (dsApSec7). As a control, we used dsRNAs against a partial sequence ( $\sim 500 \mathrm{nt})$ of the firefly luciferase gene (dsLuci) (Lee et al., 2001). Knockdown effect by dsApSec7 was confirmed by its significant blockage of the expression of ApSec7 compared with dsLuci (Fig. 7A). Injection of dsApSec7 into sensory neurons significantly blocked the induction of LTF at sensory-to-motor neuron synapses without changing the level of basal synaptic transmission, 
A
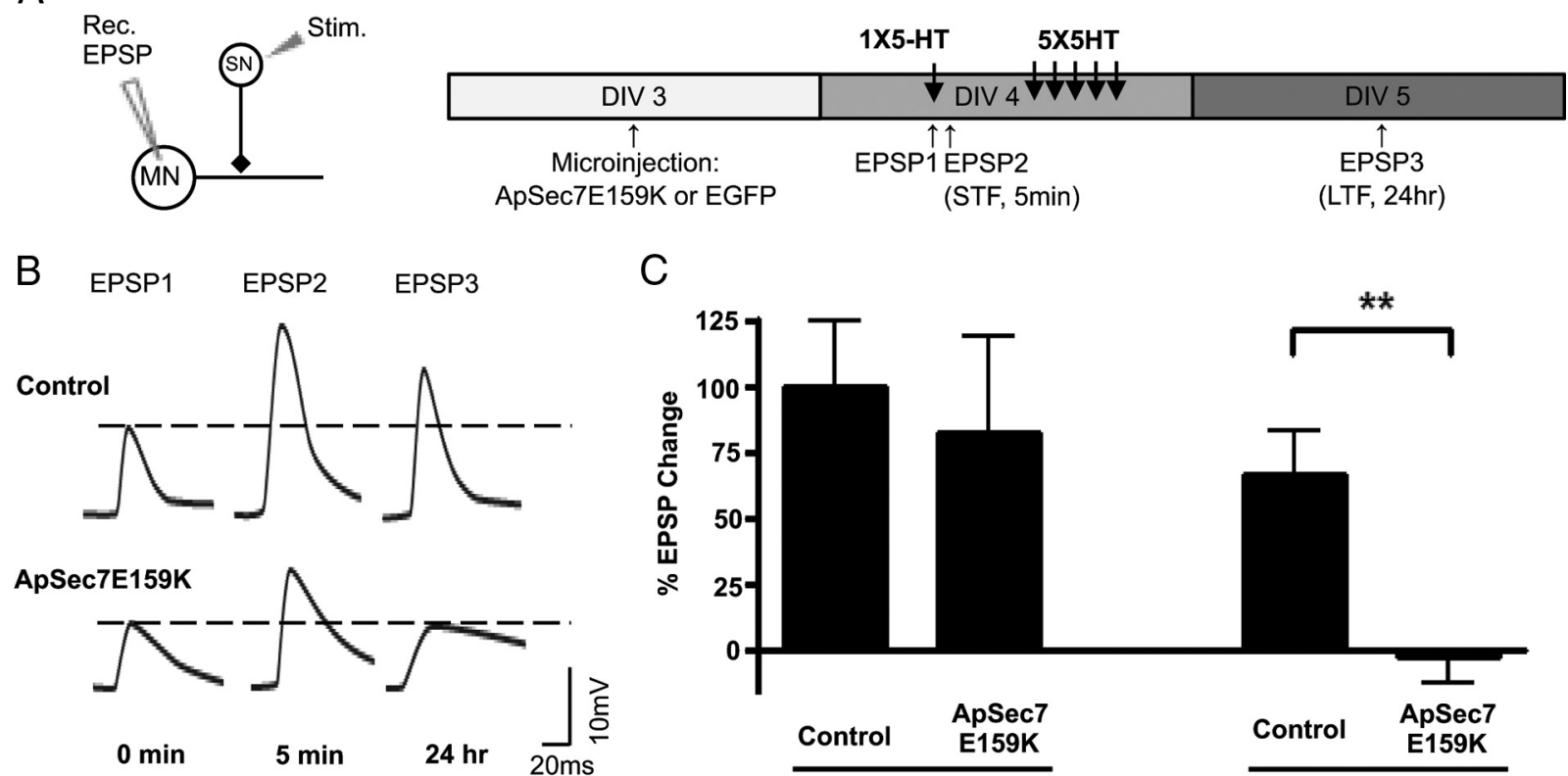

C

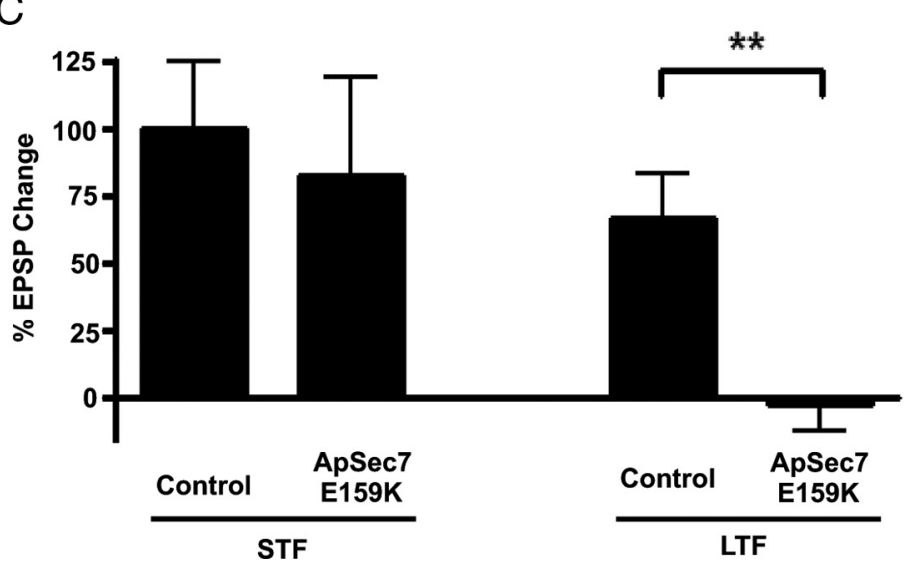

Figure 6. Perturbation of ApSec7 signaling pathway impairs the induction of LTF. A, Experimental design. Left, A schematic illustration of the intracellular EPSP recordings at the postsynaptic motor neurons (MN) in response to the extracellular stimulation of presynaptic sensory neurons (SN). Right, Daily experiments performed on the cultured neurons. DIV, Days in vitro; EPSP1, baseline measurement; EPSP2, STF induced by one pulse of 5-HT treatment for $5 \mathrm{~min}$; EPSP3, LTF measured at $24 \mathrm{~h}$ after five pulses of 5 -HT treatment. $B$, Example traces of evoked EPSP measured as shown in $A$. Control, EGFP expression in the presynaptic sensory neurons; ApSec7E159K, overexpression of ApSec7E159K with EGFP. C, Percentage change in EPSP amplitude (control, $n=11 ;$ ApSec7E159K, $n=11$; mean \pm SEM; ${ }^{* *} p<0.01$, unpaired $t$ test $)$.

whereas dsLuci-injected cells displayed normal induction of LTF (Fig. 7B).

\section{Overexpression of ApSec7 proteins} enhances synaptic growth and occludes LTF

Finally, we tested the direct effect of activating ApSec7 by overexpressing ApSec7 into sensory neurons in sensorimotor neuron cocultures. We now observed an increase in both the total number of presynaptic varicosities, as well as an increase in the amplitude of the evoked EPSPs at the synapses between sensory and motor neurons (Fig. $8 A, B)$. This effect of ApSec7 was highly similar to the effect of overexpressed msec7-1 in Aplysia neurons (Huh et al., 2003). We further found that the induction of LTF was occluded at these potentiated synapses (Fig. $8 C$ ), whereas short-term facilitation (Fig. $8 D$ ) or synaptic depression was not altered (Fig. 8E). Thus, not only is the endogenous ApSec7-mediated signaling pathway required for the induction of LTF, but also the overactivation of ApSec7 signaling can mimic the induction of LTF by promoting synaptic growth at the sensory-to-motor neuron synapse.

\section{Discussion}

In this study, we report a novel signaling pathway in Aplysia, the ApSec7/PIP5KI $\alpha$ / $\mathrm{PI}(4,5) \mathrm{P}_{2}$ pathway that regulates three of the defining features of long-term mem-
A
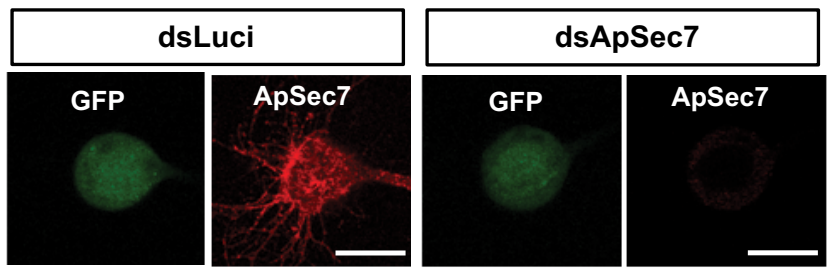

B
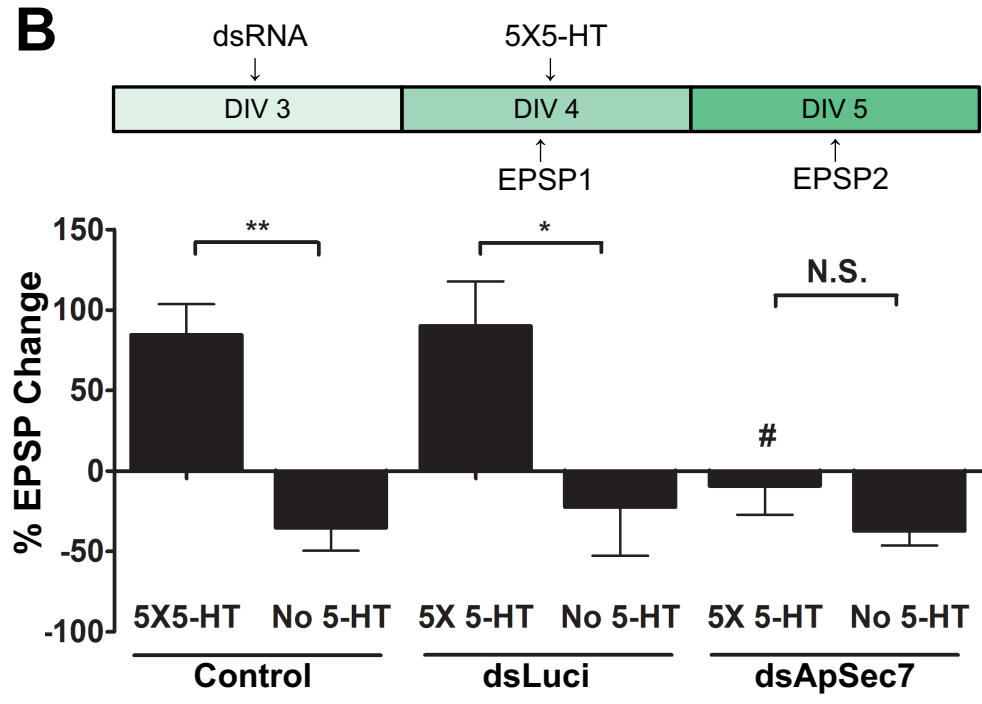

Figure 7. Knockdown of ApSec7 by dsRNA blocks LTF. $A$, Coinjected dsApSec7, but not dsLuci, blocked the expression of ApSec7 (red) in cultured sensory neurons. Scale bars, $40 \mu \mathrm{m}$. $\boldsymbol{B}$, Knockdown of endogenous ApSec7 blocks LTF significantly. Control, Without injection ( $5 \times 5-\mathrm{HT}, n=4$; No 5-HT, $n=11)$; dsLuci, control dsRNA ( $5 \times 5-\mathrm{HT}, n=5 ;$ № 5 -HT, $n=7)$; dsApSec7, dsRNA against ApSec7 (5×5-HT, $n=7 ;$ NN 5-HT, $n=11) .{ }^{*} p<0.05 ;{ }^{* *} p<0.01 ; \mathrm{NS}, p=0.20$, unpaired $t$ test; ${ }^{*} p<0.05$, one-way ANOVA followed by Dunnett's multiple comparison test. N.S., Not significant. 
A

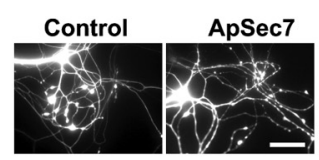

**

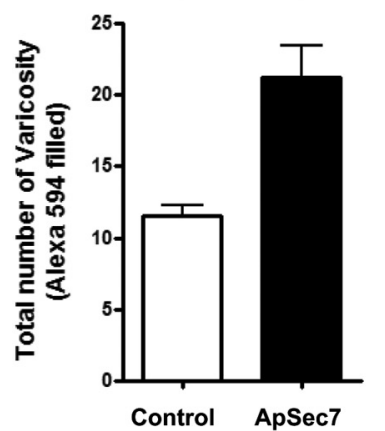

$\mathrm{D}$

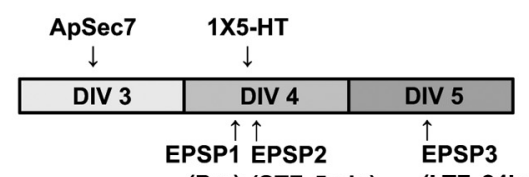

(Pre) (STF, 5min) (LTF, 24hr)
B
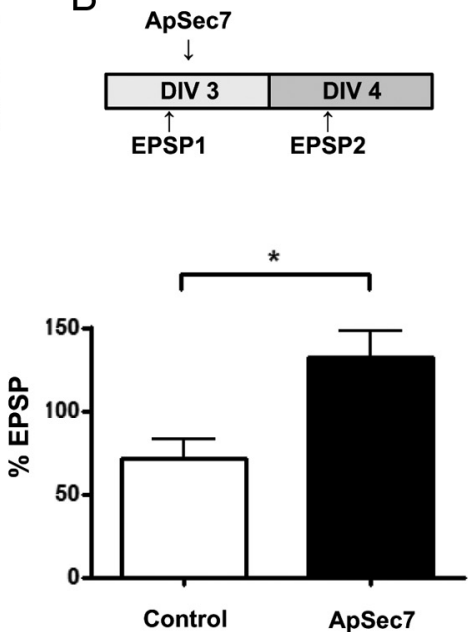

C

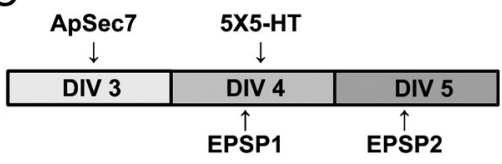

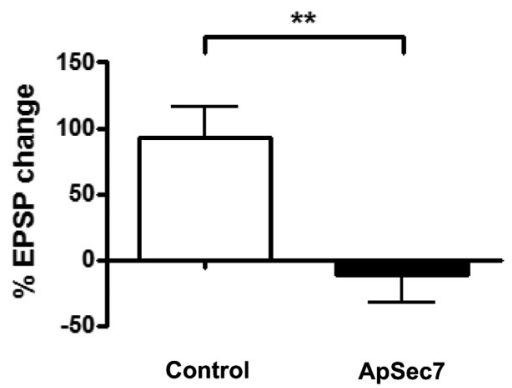

$\mathrm{E}$
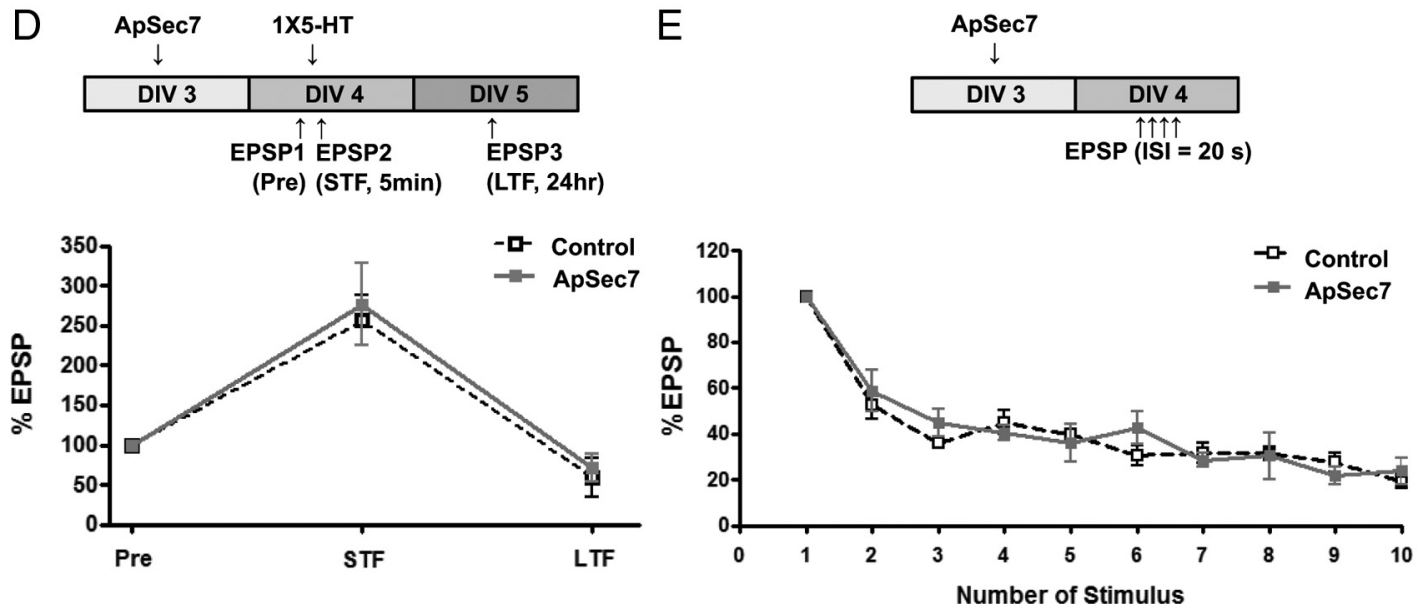

Figure 8. Upregulation of Apsec7 signaling pathway enhances synaptic growth and occludes LTF. $A$, Top, Representative images of varicosities without (Control) or with expression of ApSec7

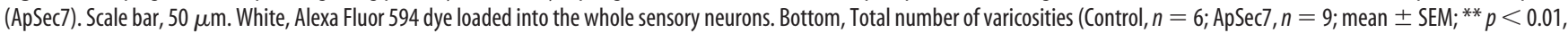
unpaired $t$ test). $\boldsymbol{B}$, Percentage EPSP measured after overexpression of Apsec7 compared with the mock injection (Control, $n=6$; ApSec7, $n=7$; mean \pm SEM; ${ }^{*} p<0.05$, unpaired $t$ test). $\boldsymbol{C}$, Percentage EPSP changes $24 \mathrm{~h}$ after five pulses of 5 -HT treatment $(5 \times 5-\mathrm{HT})$; Control, $n=7 ; \mathrm{ApSec} 7, n=7 ;$ mean $\pm \mathrm{SEM} ;{ }^{* *} p<0.01$, unpaired $t$ test. D, Percentage EPSP measured after one pulse of 5-HT treatment for $5 \mathrm{~min}$. The presynaptic sensory neuron was either overexpressed with ApSec7 ( $n=3$ ) or not expressed (Control; $n=3)$. Pre, Before 5-HT treatment; STF, 5 min after 5 -HT treatment; LTF, $24 \mathrm{~h}$ after $5-\mathrm{HT}$ treatment (mean \pm SEM). $\boldsymbol{E}$, Synaptic depression (mean \pm SEM) measured by continuous stimulation of presynaptic neurons either without (Control; $n=5$ ) or with the overexpression of $\operatorname{ApSec} 7(n=4)$.

ory storage at the sensory-to-motor neuron synapse of the gillwithdrawal reflex in Aplysia: (1) 5-HT-induced internalization of TM-apCAM, (2) learning-induced synaptic growth, and (3) long-term synaptic facilitation. The analysis of this pathway in turn has given us new insights into the mechanisms that govern learning-related internalization of apCAM and how these are coupled to the molecular changes that initiate the enduring functional and structural changes that underlie long-term memory storage.

Molecular mechanisms of the internalization of TM-apCAM and its importance for synaptic growth during LTF

Our study has characterized a novel signaling pathway that plays a critical role in the 5-HT-induced long-term synaptic plasticity at the sensory-to-motor neuron synapse in Aplysia. Not only does the ApSec7/PIP5KI $\alpha$ signaling pathway regulate the internalization of TM-apCAM, but it also is required for learning-related synaptic growth and long-term synaptic facilitation. Our findings support previous reports that TM-apCAM internalization is important for both synaptic growth and LTF (Mayford et al., 1992;
Han et al., 2004). Furthermore, we have revealed a new molecular mechanism that links TM-apCAM internalization with the 5-HT-induced growth of new synaptic connections. Regulation of structural dynamics via ApSec7/PIP5KI $\alpha$ appears to be more important for LTF, because we did not observe any changes in short-term facilitation or synaptic transmission per se by altering the activity of ApSec7/PIP5KI $\alpha$. Thus, our data suggest that the ApSec7/PIP5KI $\alpha / \mathrm{PI}(4,5) \mathrm{P}_{2}$ pathway is recruited by stimuli that induce long-term synaptic plasticity and is required specifically for the growth of new sensory-to-motor neuron synapses that accompanies both the induction and persistence of LTF.

How does activation of ApSec7/PIP5KI $\alpha$ induce the internalization of TM-apCAM during 5-HT signaling? It is plausible to think that local enrichment of $\mathrm{PI}(4,5) \mathrm{P}_{2}$ at the plasma membrane might generate an active platform for the internalization of TMapCAM. Membrane $\mathrm{PI}(4,5) \mathrm{P}_{2}$ is important for the membrane recycling and trafficking of other types of cell-adhesion molecules (Brown et al., 2001; Wong and Isberg, 2003; Zimmermann et al., 2005; Sheetz et al., 2006). An increase in the level of membrane $\mathrm{PI}(4,5) \mathrm{P}_{2}$ can recruit signaling molecules with a high affin- 
ity for the $\mathrm{PI}(4,5) \mathrm{P}_{2}$ lipid, and even $\mathrm{PI}(4,5) \mathrm{P}_{2}$ can be directly involved in the activation of downstream signaling molecules (Di Paolo and De Camilli, 2006). This leads us to suggest that 5-HT signaling can activate the ApSec7/PIP5K signaling pathway in Aplysia sensory neurons, which is required for the internalization of TM-apCAM and serves to induce the synaptic growth that accompanies LTF.

Although internalization of TM-apCAM is critical for synaptic growth, the specific nature of the structural changes recruited by the internalization of TM-apCAM remains unknown. In Aplysia, the learning-related formation of new sensory neuron varicosities is mediated by at least two processes, either (1) the budding off or splitting of preexisting varicosities enriched with synaptic proteins (Bailey and Kandel, 1993; Hatada et al., 2000; Kim et al., 2003) or (2) the outgrowth of new sensory neuron filopodia, some of which mature into functionally competent varicosities (Udo et al., 2005). A major signaling molecule that induces synaptic growth by promoting the formation of filopodia is ApCdc42 (Udo et al., 2005). However, it is unlikely that the internalization of TM-apCAM by the activation of the ApSec7/ PIP5KI $\alpha$ signaling pathway increases the number of varicosities by generating new filopodia: to begin with, we have not observed an increase in formation of filopodia on neurites when we overexpressed ApSec7 in sensory neurons despite the increase in number of varicosities (Fig. 8A). This is quite different from the activation of ApCdc42, which dramatically increases the number of filopodia (Udo et al., 2005). Second, a DN form of ApCdc42 did not suppress ApSec7-mediated neuritogenesis (Fig. 3B). This result indicates that $\mathrm{ApCdc} 42$ is not a downstream molecule of the ApSec7 signaling pathway. Finally, previous reports have found that the internalization of TM-apCAM requires PKA activity (Bailey et al., 1992; Lee et al., 2007), whereas the formation of new filopodia does not require PKA but rather requires phosphatidylinositol 3-kinase (PI3K)/PLC/ApCdc42 signaling (Udo et al., 2005). Thus, the ApSec7-mediated signaling pathway that leads to both the internalization of TM-apCAM and synaptic growth appears to be independent from the ApCdc42-mediated pathways that induce the formation of filopodia in sensory neurons. Future studies are required to determine whether the signaling pathways that lead to the internalization of TM-apCAM may also play a role in the splitting of preexisting enriched varicosities during LTF in Aplysia.

Although the major downstream molecule of ApSec7 seems to be the PIP5KI $\alpha$, other signaling pathways that regulate cytoskeletal rearrangement and membrane recycling may play a critical role in inducing the observed structural changes in Aplysia neurons. For example, actin remodeling and microtubules are known to be modulated by various types of Rho GTPases, such as GEF-H1 (Krendel et al., 2002). Because ApSec7-mediated neuritogenesis was not blocked by DN forms of Rac1 or Cdc42 (Fig. $3 B$ ), ApSec7-mediated signaling pathway may not modulate the actin cytoskeletons directly. Rather, we think it more likely that ApSec7-mediates adhesion-dependent trafficking of other membrane and cytoskeletal components, such as lipid rafts and microtubules (Balasubramanian et al., 2007), and that these may cooperate with PIP5KI $\alpha$ to play a critical role in promoting extensive neuritogenesis and structural rearrangements leading to synaptic growth. Furthermore, the increased level of membrane $\mathrm{PI}(4,5) \mathrm{P}_{2}$ and activated microtubules may affect the kinetics of synaptic vesicle release and enhance the synaptic transmission in sensory-to-motor neuron synapses (Fig. 8B). Supporting this, we have found previously that the overexpression of mutant msec7-1 (msec7-1 E157K) decreased synaptic transmis- sion without affecting the number of varicosities (Huh et al., 2003). Additional studies will be required to dissect the role of ApSec7/PIP5KI $\alpha$ pathway on the structural changes of synaptic varicosities and the synaptic vesicles that are released within a single presynaptic varicosity.

\section{Role of $\mathrm{PI}(4,5) \mathrm{P}_{2}$ in LTF: the $\mathrm{PI}(4,5) \mathrm{P}_{2}$ synthetic pathway is important for synaptic growth and the maintenance of synaptic facilitation}

Lipid $\mathrm{PI}(4,5) \mathrm{P}_{2}$ is important for regulating lipid signaling and surface trafficking of membrane proteins in a variety of cell types (Czech, 2003). In neuronal cells, $\mathrm{PI}(4,5) \mathrm{P}_{2}$ synthesis is important for the regulation of channel gating and for receptor signaling (Mathie, 2007; Prieto et al., 2011; Ying et al., 2011), and defects in $\mathrm{PI}(4,5) \mathrm{P}_{2}$ synthesis are associated with both memory loss and other neuronal diseases (Berman et al., 2008; Voronov et al., 2008; Di Paolo and Kim, 2011). Here, we have found that the $\mathrm{PI}(4,5) \mathrm{P}_{2}$ synthetic signaling pathway is also critical for the internalization of TM-apCAM in sensory neurons and the subsequent synaptic growth, thus providing a new insight into the importance of $\mathrm{PI}(4,5) \mathrm{P}_{2}$ synthesis for synaptic growth and facilitation that underlie learning and memory storage.

Not only does $\mathrm{PI}(4,5) \mathrm{P}_{2}$ directly bind to other signaling molecules, to activate or recruit them, but it is also required for subsequent production of other second-messenger molecules that are crucial for various types of cell signaling. Receptor activation of PLC results in the hydrolysis of membrane $\mathrm{PI}(4,5) \mathrm{P}_{2}$, yielding the well-known second messengers inositol-1,4,5-triphosphate and diacylglycerol (Hughes and Putney, 1988). Also, PI3K directly phosphorylates $\mathrm{PI}(4,5) \mathrm{P}_{2}$ to generate phosphatidylinositol- $(3,4,5)$ triphosphate that recruits other signaling molecules in concert with PI $(4,5) \mathrm{P}_{2}$ (Di Paolo and De Camilli, 2006). During LTF in Aplysia, the activity of both PI3K and PLC is required for the ApCdc42mediated outgrowth of filopodia and new varicosity formation (Udo et al., 2005). Although we could not observe any direct formation of filopodia by activation of ApSec7/PIP5KI $\alpha / \mathrm{PI}(4,5) \mathrm{P}_{2}$ signaling pathway (not sufficient), it is plausible that the increased level of $\mathrm{PI}(4,5) \mathrm{P}_{2}$ on the plasma membrane during stimulation with 5-HT might provide a permissive condition (necessary) for other types of synaptic remodeling and growth that are induced by different signaling molecules, such as ApCdc42-mediated filopodia formation.

In conclusion, our study suggests membrane $\mathrm{PI}(4,5) \mathrm{P}_{2}$ synthesis by ApSec7/PIP5K is a critical component of the initial molecular steps that lead to long-term synaptic plasticity in Aplysia. 5-HT stimuli recruit ApSec7 and its downstream lipid signaling pathway to initiate the internalization of TM-apCAM and the induction of both synaptic growth and LTF.

\section{References}

Bailey CH, Kandel ER (1993) Structural-changes accompanying memory storage. Annu Rev Physiol 55:397-426. CrossRef Medline

Bailey CH, Kandel ER (2008) Synaptic remodeling, synaptic growth and the storage of long-term memory in Aplysia. Prog Brain Res 169:179-198. CrossRef Medline

Bailey CH, Chen M, Keller F, Kandel ER (1992) Serotonin-mediated endocytosis of ApCAM-an early step of learning-related synaptic growth in Aplysia. Science 256:645-649. CrossRef Medline

Bailey CH, Kaang BK, Chen M, Martin KC, Lim CS, Casadio A, Kandel ER (1997) Mutation in the phosphorylation sites of MAP kinase blocks learning-related internalization of apCAM in Aplysia sensory neurons. Neuron 18:913-924. CrossRef Medline

Bailey CH, Kandel ER, Si K (2004) The persistence of long-term memory: a molecular approach to self-sustaining changes in learning-induced synaptic growth. Neuron 44:49-57. CrossRef Medline

Balasubramanian N, Scott DW, Castle JD, Casanova JE, Schwartz MA (2007) 
Arf6 and microtubules in adhesion-dependent trafficking of lipid rafts. Nat Cell Biol 9:1381-1391. CrossRef Medline

Berman DE, Dall'Armi C, Voronov SV, McIntire LB, Zhang H, Moore AZ, Staniszewski A, Arancio O, Kim TW, Di Paolo G (2008) Oligomeric amyloid-beta peptide disrupts phosphatidylinositol-4,5-bisphosphate metabolism. Nat Neurosci 11:547-554. CrossRef Medline

Brown FD, Rozelle AL, Yin HL, Balla T, Donaldson JG (2001) Phosphatidylinositol 4,5-bisphosphate and Arf6-regulated membrane traffic. J Cell Biol 154:1007-1017. CrossRef Medline

Casanova JE (2007) Regulation of arf activation: the sec7 family of guanine nucleotide exchange factors. Traffic 8:1476-1485. CrossRef Medline

Cherfils J, Ménétrey J, Mathieu M, Le Bras G, Robineau S, Béraud-Dufour S, Antonny B, Chardin P (1998) Structure of the Sec7 domain of the Arf exchange factor ARNO. Nature 392:101-105. CrossRef Medline

Coppolino MG, Dierckman R, Loijens J, Collins RF, Pouladi M, JongstraBilen J, Schreiber AD, Trimble WS, Anderson R, Grinstein S (2002) Inhibition of phosphatidylinositol-4-phosphate 5-kinase I alpha impairs localized actin remodeling and suppresses phagocytosis. J Biol Chem 277: 43849-43857. CrossRef Medline

Czech MP (2003) Dynamics of phosphoinositides in membrane retrieval and insertion. Annu Rev Physiol 65:791-815. CrossRef Medline

Di Paolo G, De Camilli P (2006) Phosphoinositides in cell regulation and membrane dynamics. Nature 443:651-657. CrossRef Medline

Di Paolo G, Kim TW (2011) Linking lipids to Alzheimer's disease: cholesterol and beyond. Nat Rev Neurosci 12:284-296. CrossRef Medline

Doughman RL, Firestone AJ, Anderson RA (2003) Phosphatidylinositol phosphate kinases put PI4,5P(2) in its place. J Membr Biol 194:77-89. CrossRef Medline

Funakoshi Y, Hasegawa H, Kanaho Y (2011) Regulation of PIP5K activity by Arf6 and its physiological significance. J Cell Physiol 226:888-895. CrossRef Medline

Glanzman DL, Kandel ER, Schacher S (1990) Target-dependent structuralchanges accompanying long-term synaptic facilitation in Aplysia neurons. Science 249:799-802. CrossRef Medline

Han JH, Lim CS, Lee YS, Kandel ER, Kaang BK (2004) Role of Aplysia cell adhesion molecules during 5-HT-induced long-term functional and structural changes. Learn Mem 11:421-435. CrossRef Medline

Hatada Y, Wu F, Sun ZY, Schacher S, Goldberg DJ (2000) Presynaptic morphological changes associated with long-term synaptic facilitation are triggered by actin polymerization at preexisting varicositis. J Neurosci 20:RC82(1-5). Medline

Hernández-Deviez DJ, Roth MG, Casanova JE, Wilson JM (2004) ARNO and ARF6 regulate axonal elongation and branching through downstream activation of phosphatidylinositol 4-phosphate 5-kinase alpha. Mol Biol Cell 15:111-120. CrossRef Medline

Honda A, Nogami M, Yokozeki T, Yamazaki M, Nakamura H, Watanabe H, Kawamoto K, Nakayama K, Morris AJ, Frohman MA, Kanaho Y (1999) Phosphatidylinositol 4-phosphate 5-kinase alpha is a downstream effector of the small G protein ARF6 in membrane ruffle formation. Cell 99:521-532. CrossRef Medline

Hughes AR, Putney JW Jr (1988) Metabolism and functions of inositol phosphates. Biofactors 1:117-121. Medline

Huh M, Han JH, Lim CS, Lee SH, Kim S, Kim E, Kaang BK (2003) Regulation of neuritogenesis and synaptic transmission by msec7-1, a guanine nucleotide exchange factor, in cultured Aplysia neurons. J Neurochem 85:282-285. CrossRef Medline

Jang DJ, Park SW, Kaang BK (2009) The role of lipid binding for the targeting of synaptic proteins into synaptic vesicles. BMB Reports 42:1-5. CrossRef Medline

Jang DJ, Park SW, Lee JA, Lee C, Chae YS, Park H, Kim MJ, Choi SL, Lee N, Kim H, Kaang BK (2010) N termini of apPDE4 isoforms are responsible for targeting the isoforms to different cellular membranes. Learn Mem 17:469-479. CrossRef Medline

Jang DJ, Lee JA, Chae YS, Kaang BK (2011) State-dependent disruption of short-term facilitation due to overexpression of the apPDE4 supershort form in Aplysia. Mol Cells 31:175-180. CrossRef Medline

Kaang BK (1996) Parameters influencing ectopic gene expression in Aplysia neurons. Neurosci Lett 221:29-32. CrossRef Medline

Kandel ER (2001) Neuroscience-the molecular biology of memory storage: a dialogue between genes and synapses. Science 294:1030-1038. CrossRef Medline
Kandel ER (2012) The molecular biology of memory: cAMP, PKA, CRE, CREB-1, CREB-2, and CPEB. Mol Brain 5:14. CrossRef Medline

Kim JH, Udo H, Li HL, Youn TY, Chen M, Kandel ER, Bailey CH (2003) Presynaptic activation of silent synapses and growth of new synapses contribute to intermediate and long-term facilitation in Aplysia. Neuron 40:151-165. CrossRef Medline

Krendel M, Zenke FT, Bokoch GM (2002) Nucleotide exchange factor GEF-H1 mediates cross-talk between microtubules and the actin cytoskeleton. Nat Cell Biol 4:294-301. CrossRef Medline

Lee JA, Kim HK, Kim KH, Han JH, Lee YS, Lim CS, Chang DJ, Kubo T, Kaang BK (2001) Overexpression of and RNA interference with the CCAAT enhancer-binding protein on long-term facilitation of Aplysia sensory to motor synapses. Learn Mem 8:220-226. CrossRef Medline

Lee SH, Han JH, Choi JH, Huh EY, Kwon YK, Kaang BK (2003) The effect of brain-derived neurotrophic factor on neuritogenesis and synaptic plasticity in Aplysia neurons and the hippocampal cell line HiB5. Mol Cells 15:233-239. Medline

Lee SH, Lim CS, Park H, Lee JA, Han JH, Kim H, Cheang YH, Lee YS, Ko HG, Jang DH, Kim H, Miniaci MC, Bartsch D, Kim E, Bailey CH, Kandel ER, Kaang BK (2007) Nuclear translocation of CAM-associated protein activates transcription for long-term facilitation in Aplysia. Cell 129:801-812. CrossRef Medline

Lee YS, Bailey CH, Kandel ER, Kaang BK (2008) Transcriptional regulation of long-term memory in the marine snail Aplysia. Mol Brain 1:3. CrossRef Medline

Loijens JC, Anderson RA (1996) Type I phosphatidylinositol-4-phosphate 5-kinases are distinct members of this novel lipid kinase family. J Biol Chem 271:32937-32943. CrossRef Medline

Mathie A (2007) Neuronal two-pore-domain potassium channels and their regulation by $G$ protein-coupled receptors. J Physiol 578:377-385. CrossRef Medline

Mayford M, Barzilai A, Keller F, Schacher S, Kandel ER (1992) Modulation of an NCAM-related adhesion molecule with long-term synaptic plasticity in Aplysia. Science 256:638-644. CrossRef Medline

Mossessova E, Gulbis JM, Goldberg J (1998) Structure of the guanine nucleotide exchange factor Sec7 domain of human Arno and analysis of the interaction with ARF GTPase. Cell 92:415-423. CrossRef Medline

Prieto GA, Perez-Burgos A, Palomero-Rivero M, Galarraga E, Drucker-Colin R, Bargas J (2011) Upregulation of D2-class signaling in dopaminedenervated striatum is in part mediated by D3 receptors acting on CaV2.1 channels via PIP2 depletion. J Neurophysiol 105:2260-2274. CrossRef Medline

Rameh LE, Tolias KF, Duckworth BC, Cantley LC (1997) A new pathway for synthesis of phosphatidylinositol-4,5-bisphosphate. Nature 390:192-196. CrossRef Medline

Raucher D, Stauffer T, Chen W, Shen K, Guo S, York JD, Sheetz MP, Meyer T (2000) Phosphatidylinositol 4,5-bisphoshate functions as a second messenger that regulates cytoskeleton-plasma membrane adhesion. Cell 100: 221-228. CrossRef Medline

Sheetz MP, Sable JE, Döbereiner HG (2006) Continuous membranecytoskeleton adhesion requires continuous accommodation to lipid and cytoskeleton dynamics. Annu Rev Biophys Biomol Struct 35:417-434. CrossRef Medline

Simons K, Gerl MJ (2010) Revitalizing membrane rafts: new tools and insights. Nat Rev Mol Cell Biol 11:688-699. CrossRef Medline

Telemenakis I, Benseler F, Stenius K, SüdhofTC, Brose N (1997) Rat homologues of yeast sec7p. Eur J Cell Biol 74:143-149. Medline

Udo H, Jin I, Kim JH, Li HL, Youn T, Hawkins RD, Kandel ER, Bailey CH (2005) Serotonin-induced regulation of the actin network for learningrelated synaptic growth requires Cdc42, N-WASP, and PAK in Aplysia sensory neurons. Neuron 45:887-901. CrossRef Medline

Voronov SV, Frere SG, Giovedi S, Pollina EA, Borel C, Zhang H, Schmidt C, Akeson EC, Wenk MR, Cimasoni L, Arancio O, Davisson MT, Antonarakis SE, Gardiner K, De Camilli P, Di Paolo G (2008) Synaptojanin 1-linked phosphoinositide dyshomeostasis and cognitive deficits in mouse models of Down's syndrome. Proc Natl Acad Sci U S A 105: 9415-9420. CrossRef Medline

Wiedemann C, Schäfer T, Burger MM (1996) Chromaffin granuleassociated phosphatidylinositol 4-kinase activity is required for stimulated secretion. EMBO J 15:2094-2101. Medline

Wong KW, Isberg RR (2003) Arf6 and phosphoinositol-4-phosphate-5kinase activities permit bypass of the Racl requirement for beta(1) 
integrin-mediated bacterial uptake. J Exp Med 198:603-614. CrossRef Medline

Yin HL, Janmey PA (2003) Phosphoinositide regulation of the actin cytoskeleton. Annu Rev Physiol 65:761-789. CrossRef Medline

Ying SW, Tibbs GR, Picollo A, Abbas SY, Sanford RL, Accardi A, Hofmann F, Ludwig A, Goldstein PA (2011) PIP(2)-mediated HCN3 channel gating is crucial for rhythmic burst firing in thalamic intergeniculate leaflet neurons. J Neurosci 31:10412-10423. CrossRef Medline

Zimmermann P, Zhang Z, Degeest G, Mortier E, Leenaerts I, Coomans C, Schulz J, N'Kuli F, Courtoy PJ, David G (2005) Syndecan recycling is controlled by syntenin-PIP2 interaction and Arf6. Dev Cell 9:377-388. CrossRef Medline 